\title{
Research on Precision Management of Diabetes Mellitus in Traditional Chinese Medicine Basing on Cloud Computing
}

\author{
Zhaohui Fang ${ }^{1}$, Ruimin $\mathrm{Lu}^{1}$, Jindong Zhao ${ }^{1}$ and Chunfa $\mathrm{Li}^{2}$ \\ ${ }^{1}$ Department of Endocrine Disease, the First Affiliated Hospital of Anhui University of Chinese Medicine, Hefei, 230021,China \\ ${ }^{2}$ Anhui Zhihong Electronic Technology Co., LTD., Hefei, 230039, China
}

\begin{abstract}
DM (diabetes mellitus) is a chronic disease caused by the body's inability to produce insulin, or by the ineffective use of the insulin produced, and it has become one of the most important non-infective diseases that threaten the public health all over the world. How to cure and manage diabetes scientifically and effectively is an important problem to be solved urgently. This paper focus on the diagnosis and treatment for DM in tradition Chinese medicine, studies the scientific management methods based on the theory of philosophy, explores the use of information technology and big data innovation method to improve the effect of diagnosis and treatment of diabetes, diabetes prevention, improves the technology and method of DM health management.
\end{abstract}

Keywords-tradition Chinese medicine; diabetes mellitus; precision treatment; cloud computing; infrastructure; big data model

\section{Diabetes And Its Present Situation}

Diabetes is one of the most important chronic non-communicable diseases which seriously threaten to global human health[1]. It is a kind of endocrine metabolic system disease, and when it becomes serious it will cause patient microvascular lesions, such as the retina, kidneys and neuropathy, and vascular lesions, such as ischemic heart disease, stroke, and peripheral vascular disease.

Most diabetics live in the low and middle income countries, and the quantity of diabetics is rapidly rising, so the treatment of diabetes has become a huge and deepening global problems. Due to the long course of diabetes, too much complications and high morbidity, diabetes brings huge burden for individuals, families, and countries, and it has become an important public health problem all over the world. Therefore diabetes is not just a simple health problem, how to solve the problem of diabetes needs various concrete policies and actions from many aspects of modern society[2].

Although the number of diabetes patient is fabulous now, but the cause and mechanism of diabetes is still not completely clear. There is no cure for diabetes patients, but some drugs can only be used to control their condition, such as insulin by injection and drugs. Generally diabetes patients can eat a lot of western medicine with side effects which will lead to loss of activity of pancreatic islets. If a patient takes excessive or inadequate drugs, his blood sugar will be unstable. And after a long time the drugs will be ineffective, so he has to change other drugs with more side effects.

As we know, western medicine and Chinese medicine in the treatment of diabetes have their own advantages[3]. For example, western medicine has the advantage of hypoglycemic action, that is to say, a few kinds of Western medicine hypoglycemic drugs are clear in insulin action mechanism clearly, suitable for relative patients, forbidden for other patients, and achieve great hypoglycemic effects.

What are the advantages of traditional Chinese medicine? Chinese medicine stresses the theory that man is an integral part of nature in regulating body, and it pays attention to the harmony between man and nature. In nature, the life is changing with the change of nature, such as some plants are only suitable for living in the south and they cannot be survival in the north. For patient individual, nature should conform to this situation is advantageous to adjust the body, this is one of the characteristics of traditional Chinese medicine.

From the concept of time, the life of Chinese people from birth to death has been divided into growth, strong, old stage, and the features are changing in each stage of life. During Chinese medicine the organ of children is not the same with the middle-aged, and deficiency of liver-yin and kidney-yin will be showed obviously when he goes to the old age. So it is beneficial to people's health by treating people differently from time to time.

Also each patient will have a different body constitution, which is actually obvious in the child stage, or the young stage. From traditional Chinese medicine, if you have the body constitution deviation, you should pay attention to adjust the physical condition in the early days to avoid being a diabetic patient.

So this paper focus on the effective treatment and better prevention for DM in traditional Chinese medicine.

\section{Research on Diabetes Health Management Mode}

How to effectively control diabetes disease and execute scientific management of public health is one of most urgent tasks to improve people's well-being. Since the beginning of the century, the community health service has prospered with the rapid economy development and national investment in public health project in China. Many community health 
service institutions are currently focusing on treatment of diabetes. However their management content, management style, management means and management program vary wildly, so there is no uniform standard of diabetes community management model which causes diabetes data not being shared to continuously improve treatment effect.

Management science is a discipline and technology which is widely used in any corner of life. At the same time, scientific research methods are very important in the study of management. First of all, the scientific method is objective, it is based on the fact which makes our study is reliable. Secondly, the scientific method is empirical, it dependents on the test of practice information so that different people in different location and different time can achieve the same conclusion by using the same method. Thirdly, the scientific method is normative, its procedures and steps of the research are orderly, clear and structured. In the end, the scientific method is more general, so the result of the scientific method is more extensive.

Therefore, it has become extremely essential and urgent to explore scientific management mode for diabetes, to set up scientific research methods, to form an effective management model, to develop diabetes standardized management, efficient control and integrated management by using the basic knowledge and basic principle of management, principles, techniques and methods.

The new model of precision health management for diabetics mainly integrates the resource of hospital, community and family to explore comprehensive prevention and control of diabetes. By setting up data sharing and medical resources sharing of regional health information system, using big data diagnosis and treatment methods, the cognitive ability and treatment level in diabetics for general practitioners at the grass-roots will be improved greatly.

\section{Research on Cloud Platform Based On Big Data}

With the popularization of network application, the network information and the amount of service are increasing gradually. At the same time, the massive data storage, the heterogeneous data distribution and so on. Cloud computing as a new technology to solve this problem arises at the historic moment.

At present, the medical data mainly comes from the basic health services (township hospital community, etc.) and professional health service institutions (hospitals, centers for Disease Control and prevention, first aid center). But the data in the present time has isolated characteristics. The isolated island data refers to the isolated, scattered business data which cannot share with each other. There are a lot of isolated data in the construction of medical information.

At present, various medical institutions (hospitals, clinics, community health centers) all have electronic health records system, which can mainly do health statistics for residents of the region to provide relevant personnel statistics service and others. But these health records systems are developed by themselves, and the data is established in a fixed server, so they generally cannot contact with the outside world. It is common that a person in different medical institutions is more likely to have multiple copies of such data. These data waste a large amount of system resources, and the utilization rate of the data is very low.

HIS is the hospital information management system, and its main task is to facilitate the management of all kinds of things within the hospital, such as patient charges management surgical management, medicine management, material management, etc. Most hospital has its own HIS system for its internal affairs, so every hospital is different, this leads to different HIS system and its data cannot be connected and shared by others.

LIS and PACS system is a relatively advanced hospital information system, which is mainly responsible for the management of the patient's experimental data, including the patient's personal information, experimental data, X-ray slice, etc. At the same time, these data generally is stored in the hospital internal server, when the patient goes to another hospital, he usually needs to do check-up again. It results in a large number of human and material being unable to realize the sharing of resources.

At present, there are a large number of health examination data in various medical institutions. Besides LIS and PACS data, the health examination data is used only for internal staff in the hospital medical. In fact, there is not much relationship between the health examination data and the hospital and its services.

In addition to the isolated data mentioned above, there are a lot of other data, such as the medical records in Centers for Disease Control, etc.

The isolated data occupy a large amount of storage resources, but they can not interconnected and shared so as to achieve effective access. Although the data utilization is very low, the hospital and patients have to pay much with great economic pressure.

So how to make sufficient use of the existing data and future data in all kinds of medical institutions to better support for doctors and managers to provide better medical service in low price for patients is the focus of this article. And we intend to build an integrated information sharing and collaborative working platform for relative medical institutions, its core is data centers which should be effectively sharing for at all levels of hospital, community medical institutions, administration of traditional Chinese medicine and other departments and cloud computing technology[4,5] should be adapted for full use of the existing physical resources and software resources

\section{A. Cloud Platform Construction}

The core of precision treatment and management platform for diabetes is to build a regional integration information sharing and collaborative work system for diabetes health management, the overall architecture is shown in figure 1.

In this platform, cloud computing technology is used in data storage, data computing and service architecture and so on. And health data acquisition, safe data transmission storage, big data analysis can be completed in this platform to maximize the implementation of health resources sharing and real-time 
management, to establish a unified service and management platform for the residents, medical institutions, government

agencies and other service objects.

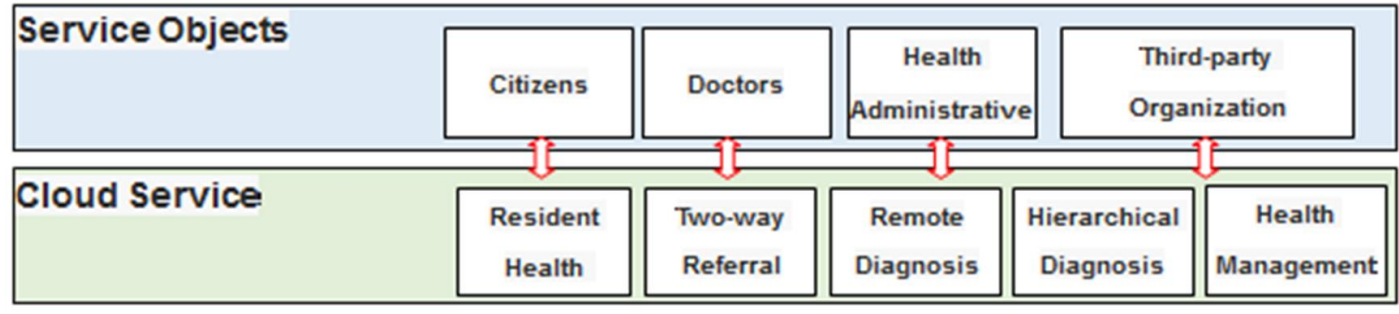

\begin{tabular}{|l|c|c|}
\hline $\begin{array}{l}\text { Cloud } \\
\text { Computing Center }\end{array}$ & $\begin{array}{c}\text { Health } \\
\text { Archive }\end{array}$ & $\begin{array}{c}\text { Electronic } \\
\text { Medical }\end{array}$
\end{tabular} \begin{tabular}{c}
$\begin{array}{c}\text { Public Health } \\
\text { Data }\end{array}$ \\
\hline
\end{tabular}
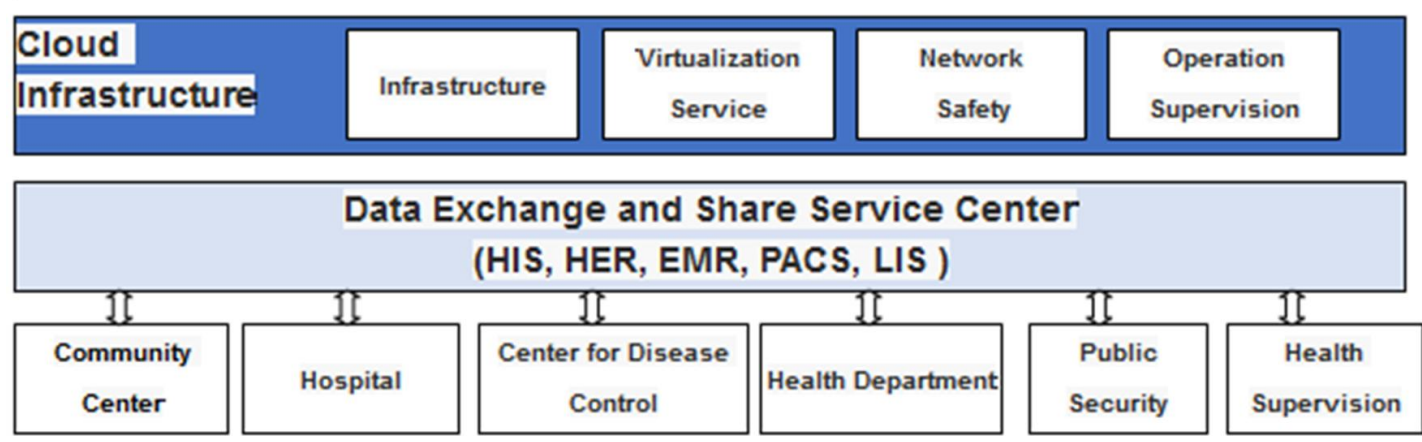

FIGURE I OVERALL ARCHITECTURE DIAGRAM OF PRECISION MANAGEMENT CLOUD PLATFORM

\section{B. Technical Solution}

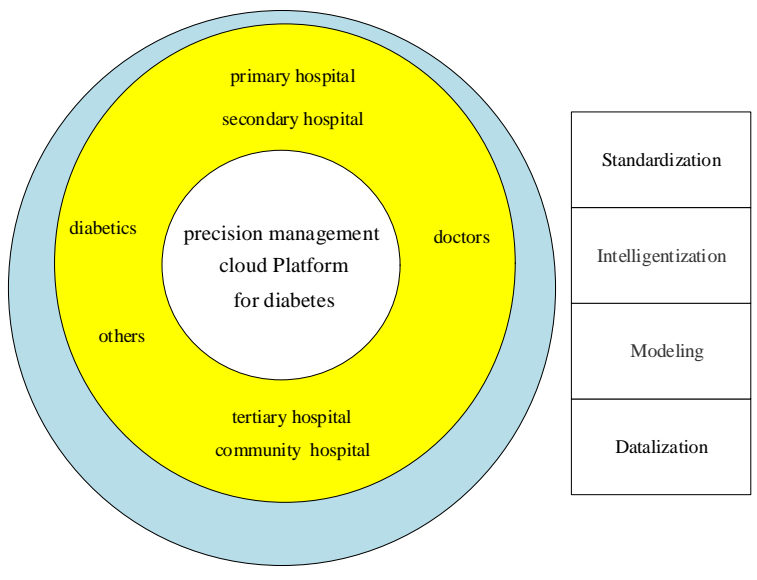

FIGURE II PRECISION TREATMENT AND MANAGEMENT APPLICATION SYSTEM FOR DIABETES

As shown in figure 2, the precision treatment and management platform for diabetes to build a health management union of Chinese medicine in Anhui province, and the hospital, community and family are integrated into an organic whole in the union. Anhui province hospital of traditional Chinese medicine (national clinical research base) is the data center, and it plays the role of technical guidance, scientific research management, training, and so on. As a primary hospital, it is responsible for technical support and scientific research management. The secondary hospital is responsible for standard diagnosis and treatment and health management. The tertiary hospital is responsible for treatment of difficult and severe diseases in diabetes. The community hospital is responsible for the services contracted and behavior intervention.

1) To set up population screening model. The task of platform is to collect diabetics information in structured records first, to establish risk evaluation model for high-risk diabetics groups, pre-diabetes and diabetes, to set upknowledge base of intervention measures, to explore standard solution for the prevention and control of diabetes in traditional Chinese medicine clinical, to realize individual behavior intervention and treatment guidelines for prevention of diabetes as early as possible.

2)To set up intelligent evaluation model. The integration collaboration network platform of hospital-community-family is effective for health management of people at high risk of diabetes and diabetics, useful for implementation of health information acquisition, analysis, intervention and evaluation, easy to optimize interventions according to the evaluation results, and viable to achieve dynamic management of diabetes.

3) To establish a hierarchical diagnosis and two-way referral model. With the aid of remote expert service, remote consultation, remote check and image, remote pathological diagnosis from the superior hospital resources, patients also enjoy big hospital expert medical services at home. It ensures medical service quality at the grass-roots level and keeps the trust from diabetics at the grass-roots level. According to priorities and the rules and standards of two-way referral system, diabetics can be guided in the orderly flow to ensure the continuity of medical services. It is of great importance for 
avoiding seek treatment cure blindly, reducing the hospital cost, and implement medical pattern classification.

4) To establish a new kind of management mode by combination of Chinese and western medicine for diabetes. The platform explores the technical implementation using Chinese and western medicine for diabetics, and its results provide experience and services for deepening the medical and health system reform and establishing the classification treatment system.

\section{Software Implementation}

The precision treatment and management platform for diabetes is implemented by constructing the general hardware platform, the cloud software and mobile Apps to connect patients, doctors and medical resources in different time and different space into an integration so as to maximize the sharing of resources in the platform.
All kinds of service software automatically connect medical equipments to the health services cloud platform[6]. After data acquisition, diabetic medical data are automatically uploaded to the cloud platform and stored in it. And then the cloud platform is constantly monitoring various data and performing intelligent analysis to push analysis report and prevention advice to the patients. At the same time, mobile phone apps establish real time communication network between doctors and patients to communicate online or offline for timely tracking, communication and interaction during the diagnosis and rehabilitation process. The software can provide patients with information collection, health management, guiding medication, or even diet and exercise of one-stop service, and provide the best solution for diabetes health management.

D. Data Analysis

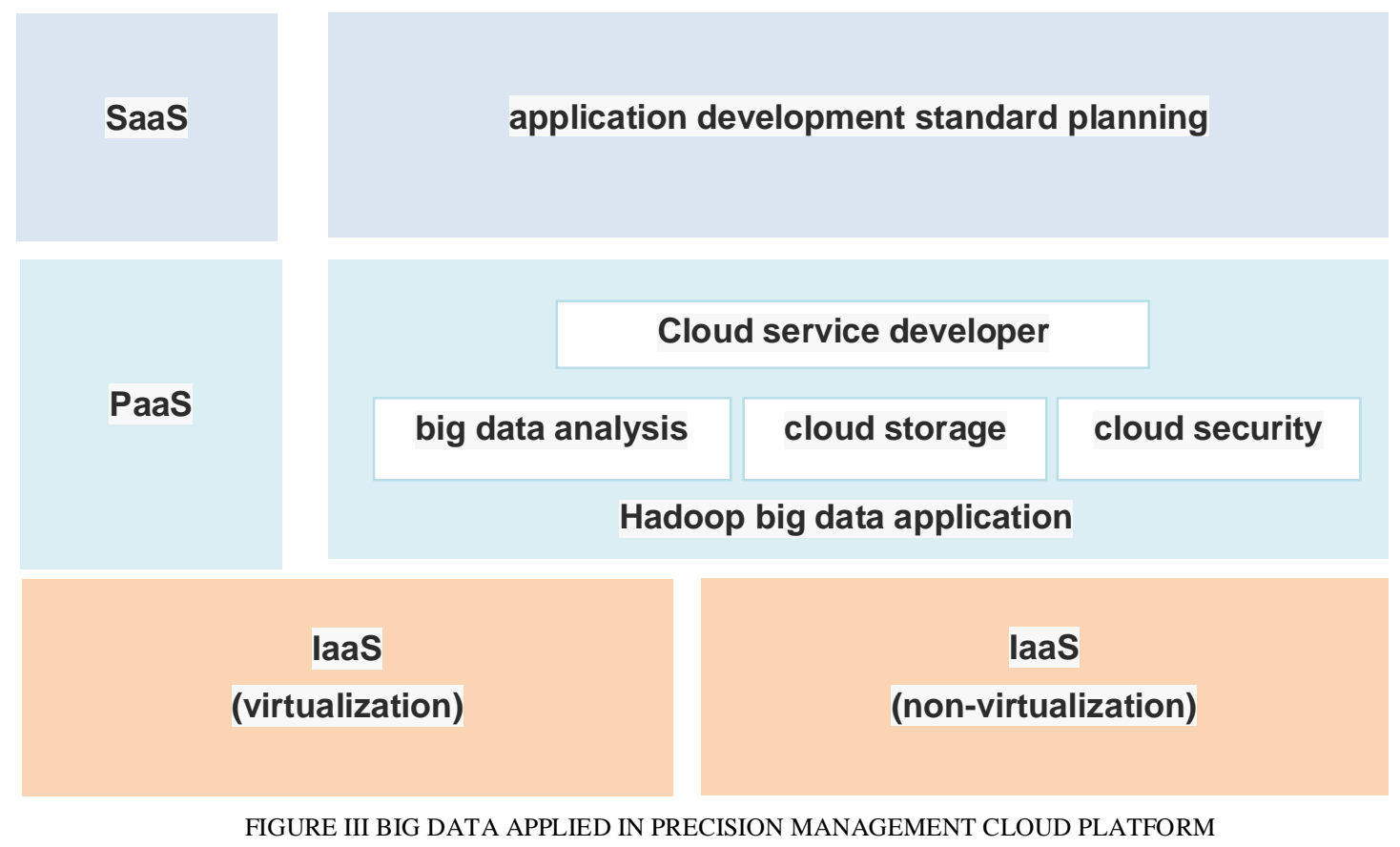

As shown in figure 3, the precision treatment and management platform is running basing on a big data center[7] using virtualization technology, resource scheduling technology, data integration, system architecture, etc., for a variety of public health resources reasonable scheduling and cooperation to make full use of resources, reduce waste of resources, improve the utilization rate of hardware and software. Virtualization technology can be used to realize the logic of the underlying infrastructure[9], to avoid all kinds of problems for physical resource deployment in the process of platform construction. Resource scheduling strategy based on virtualization technology can realize real-time scheduling of resource request. And the virtualization cloud computing architecture can make developers to use the simplest way to achieve the complex function in the system.

Big data analysis with Hadoop technology can achieve tens of thousands of data import and query[8], so every query can be done in second response. Distributed data warehouse and mining engine can double statistical efficiency. Archival data can be freely expanded to realize coexistence of old and new data and to support the expansion of business. Distributed storage and redundancy is used to improve data reliability, ensure a reliable and long-term preservation for medical archives. Non-stop expansion is supported to improve storage capacity and computing power.

The precision treatment and management platform can obtain a great deal of annual information of diabetes patient quantity statistical, ages ratio statistics, the number of patients with occupational statistics according to region. As the increasing number of people with diabetes the platform have enough patients clinical trial data to construct the corresponding data analysis model which is more accurate and reliable to find what factors are closely associated with diabetes, and the best precision prevention program for 
different diabetes patients.

\section{CONCLUSION}

Medical research clearly shows that, in addition to genetic factors of diabetes, diabetes mellitus is closely related to unhealthy lifestyle such as unreasonable diet, lack of exercise, smoking, etc. As a result, it is better to strengthen the exercise and control diet to avoid diabetes mellitus.

E-Health is defined by the World Health Organization as the use of information and communication technologies for health. These technologies can be used to deliver programs and resources and supports via a range of modalities including the Internet, mobile devices and the telephone[10]. How can new technologies improve the management of diabetes in traditional Chinese medicine. This paper highlights how these new technologies can be used to improve the prevention and management of diabetes, in particular, by really helping diabetes patients to improve their self-management of diabetes.

The precision treatment and management cloud platform focus on diabetes patients which provides file establishment and management, treatment management, follow-up visit management and drug management for diabetes patients. The platform takes knowledge as center, establishes diabetes diet, exercise, medication knowledge base, provides such service as prevention and control with diabetes, reasonable diet and others with mobile terminals.

At present, a total of 124 hospital institutions at all levels join in the precision treatment and management cloud platform, a total of 65757 diabetes archives have been established, a total of 46237 effective follow-up cases have been recorded. The establishment and application of the platform is of great significance to explore the new diabetes health management model, to develop the diabetes health management, to improve the ability of prevention and cure of diabetes, to guarantee public health and social security.

\section{ACKNOWLEDGMENT}

The authors acknowledge the National Chinese Medicine Clinical Research Foundation of China (Grant: JDZX2015125). Base: Anhui province Chinese medicine hospital.

\section{REFERENCES}

[1] Zeng Xiao-hua, Guo Wen-ming. Design and realization of post-discharge diabetes patient health monitoring and management system based on cloud platform[J]. Chinese Medical Equipment Journal,2015,36(4):56-59

[2] Zhao Qiu-fen, Huang Yue-shan, Wu Xiao-ming. Research of Community Health Service Platform Based on .NET[J]. Chinese Medical Equipment Journal,2013,34(4):50-52

[3] Min Jie, Zhu Dezeng. Design and Implementation on Community Health Care Diabetes Comprehensive Management System of Integrated Chinese and Western Medicine[J]. Chinese Medical Records, 2014,15(7):44-47

[4] Ramachandran M. Advances in cloud computing research[M]. 2014.

[5] Bento A M, Aggarwal A. Cloud computing service and deployment models: layers and management[M]. Hershey, PA: Business Science Reference, 2013. 368.
[6] Foster I, Vasiliadis V.Saa S for science: the path to reality for research in the cloud[Z].Chicago, Illinois: ACM, 2012.

[7] Cusumano M. Cloud computing and SaaS as new computing platforms[J]. Commun. ACM,2010, 53(4): 27-29.

[8] Tom White. Hadoop:The Definitive Guide[M]. O'Reily Media, 2012

[9] Tan C H, Teh Y W. Synthetic Hardware Performance Analysis in Virtualized Cloud Environment for Healthcare Organization[J]. Journal of medical systems, 2013, 37(4): 1-13

[10] Hansen, J. Cloud to Device Push Messaging on Android: A Case Study[C]. 26th International Conference on Advanced Information Networking and Applications Workshops. 2012:1298-1303. 